BMJ Open

Diabetes

Research

\& Care

\title{
Adherence to ophthalmology referral, treatment and follow-up after diabetic retinopathy screening in the primary care setting
}

\author{
George Bresnick (D) , 1,2 Jorge A Cuadros (D) ,,2 Mahbuba Khan (D) , \\ Sybille Fleischmann (D) , ${ }^{4}$ Gregory Wolff (D) , ${ }^{4}$ Andrea Limon, ${ }^{2}$ Jenny Chang (D) , \\ Luohua Jiang (D) , ${ }^{6}$ Pablo Cuadros (D) , ${ }^{2}$ Elin Rønby Pedersen (D) ${ }^{7}$
}

To cite: Bresnick G, Cuadros JA, Khan M, et al. Adherence to ophthalmology referral, treatment and follow-up after diabetic retinopathy screening in the primary care setting. BMJ Open Diab Res Care 2020;8:e001154. doi:10.1136/ bmjdrc-2019-001154

- Additional material is published online only. To view please visit the journal online (http://dx.doi.org/10.1136/ bmjdrc-2019-001154).

A modified abstract of this manuscript was accepted for presentation at the annual meeting of the Association for Research in Vision and Ophthalmology in Baltimore, MD, from 3 to 7 May 2020.

Received 2 January 2020 Revised 7 May 2020 Accepted 24 May 2020

Check for updates

(c) Author(s) (or their employer(s)) 2020. Re-use permitted under CC BY-NC. No commercial re-use. See rights and permissions. Published by BMJ.

For numbered affiliations see end of article.

Correspondence to Dr George Bresnick; visionforall2020@gmail.com

\section{ABSTRACT}

Introduction Telemedicine-based diabetic retinopathy screening (DRS) in primary care settings has increased the screening rates of patients with diabetes. However, blindness from vision-threatening diabetic retinopathy (VTDR) is a persistent problem. This study examined the extent of patients' adherence to postscreening recommendations.

Research design/methods A retrospective record review was conducted in primary care clinics of a large county hospital in the USA. All patients with diabetes detected with VTDR in two time periods, differing in record type used, were included in the study: 2012-2014, paper charts only; 2015-2017, combined paper charts/electronic medical records (EMRs), or EMRs only. Adherence rates for keeping initial ophthalmology appointments, starting recommended treatments, and keeping follow-up appointments were determined.

Results Adequate records were available for 6046 patients; 408 (7\%) were detected with VTDR and recommended for referral to ophthalmology. Only $5 \%$ completed a first ophthalmology appointment within recommended referral interval, $15 \%$ within twice the recommended interval, and $51 \%$ within 1 year of DRS. Patients screened in 2015-2017 were more likely to complete a first ophthalmology appointment than those in 2012-2014. Ophthalmic treatment was recommended in half of the patients, of whom $94 \%$ initiated treatment. A smaller percentage $(41 \%)$ adhered completely to post-treatment follow-up. Overall, $28 \%$ of referred patients: (1) kept a first ophthalmology appointment; (2) were recommended for treatment; and (3) initiated the treatment. Most patients failing to keep first ophthalmology appointments continued non-ophthalmic medical care at the institution. EMRs provided more complete information than paper charts.

Conclusions Reducing vision impairment from VTDR requires greater emphasis on timely adherence to ophthalmology referral and follow-up. Prevention of visual loss from VTDR starts with retinopathy screening, but must include patient engagement, adherence monitoring, and streamlining ophthalmic referral and management. Revision of these processes has already been implemented at the study site, incorporating lessons from this investigation.

\section{Significance of this study}

What is already known about this subject?

- Regular diabetic retinopathy screening (DRS) is essential for the early detection of vision-threatening diabetic retinopathy (VTDR).

- The timely treatment of patients found to have VTDR can prevent severe vision loss in up to $90 \%$ of patients; delays in needed treatment can result in unnecessary and irreversible vision loss.

What are the new findings?

- A low proportion of patients detected with VTDR after DRS, and recommended for referral to ophthalmology, attended a first ophthalmology visit within the recommended time frame.

- Most patients who did attend an ophthalmology visit and were recommended for treatment, initiated that treatment, but a substantially smaller percent adhered completely to post-treatment follow-up.

- The majority of patients who failed to adhere to these recommendations were still receiving nonophthalmic medical care within the same institution.

How might these results change the focus of research or clinical practice?

- The primary care team must be able to monitor efficiently the adherence of patients' keeping a first ophthalmology appointment within the recommended time frame.

- Research is required to develop more efficient monitoring, and interventions to improve adherence to specialty referral, treatment and follow-up.

\section{INTRODUCTION}

Telemedicine-based diabetic retinopathy screening (DRS) programs in the primary care setting have become widespread, ${ }^{1}$ and have been successful in improving screening rates for retinopathy among patients with diabetes. ${ }^{2-4}$ Digital retinal imaging with a nonmydriatic camera can be effective in identifying individuals with vision-threatening 
diabetic retinopathy (VTDR) who may need treatment of their eyes to prevent serious vision loss. ${ }^{5}$ However, to be most effective in preventing vision loss, screening must also be coupled with timely referral of patients with VTDR to eye specialists and with the administration of appropriate ocular therapy. To accomplish this when DRS is conducted in the primary care setting, the nonspecialist must educate the patient about the risks of VTDR and the importance of seeking timely eye specialist intervention. ${ }^{6}$ Extrapolation of data from randomized clinical trials of laser treatment for proliferative diabetic retinopathy (PDR) estimates that the rate of blindness from untreated severe PDR can be reduced by as much as $90 \%$ through early detection and prompt treatment before vision loss develops. ${ }^{7}$

Despite overall improvement in screening rates nationwide, diabetic retinopathy remains the leading cause of blindness among working age adults in the USA. ${ }^{8} \mathrm{~A}$ factor contributing to this may be a breakdown and inefficiency in the process for eye specialty referrals, treatment recommendations and follow-up appointments. ${ }^{9}$ There is generally no comprehensive system for tracking patients referred from primary care settings to ophthalmology, and very few studies have been published on this topic. From those that have been published, it appears that many patients with VTDR are not being optimally treated..$^{10-12}$

To understand the relationship between DRS-generated ophthalmology referral and ocular treatment for VTDR, a retrospective record review study was conducted in a large county medical center, where primary care-based DRS had been successfully implemented since 2011. Data from 2012 to 2017 were collected and analyzed.

\section{RESEARCH DESIGN AND METHODS}

\section{Patient identification and record reviews}

The study was conducted in the Family Medicine Department at the Riverside University Health System-Medical Center (RUHS-MC), Riverside, California. Following Institutional Review Board approval, all patients who had received telemedicine-based DRS ${ }^{13}$ in the Family Care Center (FCC) at RUHS-MC, over the time periods specified below, were identified in the screening database. The patients had been referred for DRS either from the FCC itself, from the Internal Medicine Clinic at RUHS-MC, or from satellite community health centers. All patients with a diagnosis of diabetes mellitus or pre-diabetes were recommended for an annual DRS, unless they had seen an eye care specialist within the previous 2 years.

The retinal images had been evaluated remotely by two experienced certified retinal graders using a standardized structured grading protocol. ${ }^{13}$ The same consultants evaluated the retinal images over the entire study period. All patients with a screening diagnosis VTDR were identified in the DRS database. VTDR was defined as one or more of the following in at least one eye: (1) PDR; (2) severe nonproliferative diabetic retinopathy (SNPDR); (3) clinically significant macular edema (CSME). Only patients recommended for referral to ophthalmology were included in the record review. The referral included a recommended referral interval, the time duration within which the patient should be seen, as determined by the retinopathy severity; the recommended referral intervals were consistent with published clinical guidelines. ${ }^{14}$ The default referral intervals were: SNPDR, 3 months; PDR, 1 month; CSME, 1 month. Certified graders could over-ride the defaults, but rarely did, except for patients with advanced PDR who were referred within 2 weeks or less. All patients were referred internally to the ophthalmology clinic in the same building as the FCC. Most were initially evaluated in the general eye service and referred to the retina specialty service if treatment was anticipated.

The medical record reviews were divided into two time periods, based on the type of medical records in use during the screening and subsequent follow-up periods. (The RUHS switched from paper charts to an electronic medical record (EMR) system in October 2016.) These were designated time period A: 2012-2014, paper charts only; time period B: 2015-2016, combined paper charts and EMRs, and 2017, EMRs only. Data were analyzed separately for the two time periods, to look for: (1) trends in screening and in referral adherence over time; (2) differences in the information obtainable from the two record types; and (3) evidence that the EMR improved adherence with referral fulfillment, as has been reported elsewhere. ${ }^{15}$

The following data were extracted for each patient: (1) date of keeping first ophthalmology appointment; (2) ocular treatment recommendation status; (3) date of initiating recommended treatment; and (4) dates of keeping subsequent treatment episodes and/or follow-up visits.

\section{Chart review interval and definition of adherence indicators}

1. The 'chart review interval' was the time between the date of a given event (eg, DRS) and the last date the records were reviewed for each patient. The 'overall chart review interval' was calculated from the date of the DRS. The minimum chart review intervals required to include a specific adherence rate in the study analysis were: (1) keeping first ophthalmology appointment, 12 months after DRS; and (2) treatment and post-treatment follow-up, 12 months after first ophthalmology appointment. Patients with shorter chart review intervals for a given measure were designated 'indeterminate' for that measure.

2. 'Adherence to keeping a first ophthalmology appointment' was the proportion of patients showing a kept appointment within a specified 'adherence interval'. The 'adherence interval' was the length of time between the DRS and first visit to the ophthalmology clinic.

3. 'Adherence to initiating treatment' was the proportion of patients initiating treatment after the recommendation was made.

4. 'Adherence to post-treatment follow-up' was defined in four categories: (1) 'non-adherent': no follow-up 
visits after initial treatment session; (2) 'partially adherent': returned for some follow-up visits, but failed to return for one or more visits within 6 months of the recommended follow-up interval; (3) 'completely adherent': returned for all follow-up visits within 6 months of the recommended intervals; and (4) 'indeterminate': could not determine due to missing data or to an inadequate chart review interval.

5. 'Adherence to follow-up Ophthalmology Clinic visits among patients not recommended for treatment' was defined using the same categories as adherence to post-treatment follow-up.

\section{Additional analyses}

The screening database was searched for repeat screening to determine whether patients failing to keep a first ophthalmology appointment within 1 year of the DRS were 'recaptured' for re-evaluation and possible re-referral. The date of repeat DRS, diagnosis, ophthalmological referral and adherence to first ophthalmology appointment were recorded. Also, the records were searched for patients who died after the DRS.

\section{Statistical analyses}

Patients' demographic variables including age, gender, ethnicity and insurance type were collected from paper charts and/or EMRs. Clinical characteristics such as years of diabetes, comorbid conditions, diabetes type and HbAlc value were also collected at or close to baseline screening.

Descriptive statistics of the study population are listed in table 1. $\chi^{2}$ tests were used to test differences of characteristics in two time periods. Kaplan-Meier curves and $\log$ rank test were performed to compare two time periods for keeping ophthalmology appointments. Outcome of time-to-event analysis was the time between screening until the date of the earliest of the following events: having a first ophthalmology appointment, or last chart review date. Patients who did not have ophthalmology appointments were included as censored observations.

Additionally, bivariate and multivariate analyses were performed to test whether patient-related factors correlated with the adherence rate for keeping a first ophthalmology appointment. The results are reported in online supplementary appendix B. A multivariate Cox proportional hazards model was fitted to estimate the HRs of keeping first ophthalmology appointments for the various patient characteristics. Analyses were performed using SAS V.9.4 (SAS Institute). Statistical significance was set at $\mathrm{p}<0.05$ with two-tailed tests.

\section{RESULTS}

Some of the major findings in the study are illustrated in the flow chart (figure 1) and are described in detail below.

\section{Baseline characteristics of study population}

A total of 3064 patients were screened in time period A, of whom six were eliminated because medical records were not available. Among those remaining, 147/3058 (5\%) were recommended for referral for VTDR; a greater proportion was referred in time period B, 2991 screened, 3 eliminated, and 261/2988 (9\%) referred $(p<0.001)$. Since the retinal image grading criteria did not change over time, the reason for the increase in referrals for time period B was inapparent. The same readers graded the images over the entire duration of the study.

The distribution of the characteristics of the referred study population, subdivided by time period, is shown in table 1. Differences of distributions between time periods were tested with $\chi^{2}$ test or Fisher's exact test.

The two time periods showed statistically significant differences as follows:

Age group: Time period B had a higher proportion of patients in the oldest age group (60+) and fewer in the youngest group (18-49).

Ethnicity: Hispanic patients formed the largest ethnic group, and this preponderance was greater in time period B.

Retinopathy severity: There was a higher percentage of patients with PDR in time period A, and a higher percentage of the milder retinopathy categories in time period B.

CSME: CSME was present in the majority of patients $(81.1 \%)$, and was more prevalent in time period B.

Primary care site: Of patients referred for DRS from the Internal Medicine Clinic, a higher percentage was referred in time period $\mathrm{B}$, and of the satellite clinics a higher percentage was referred in time period A. There was little difference in the percentage of patients referred from the FCC between each time period.

Insurance type: The most common types of insurance in descending order were MediCal, Medicare, and private; close to $10 \%$ of patients were covered by the Medically Indigent Services Program (MISP). (This is a safety net program sponsored by Riverside County, and designed to cover 'medical care to prevent disability'. It is not, strictly speaking, insurance, but is treated as such for the purpose of this analysis.) Other types included: uninsured, charity patients, and self-pay. For slightly more than $25 \%$ of patients, the insurance status could not be determined from the records.

Insurance category: There was a much higher percentage of insured patients in time period $\mathrm{B}$ and a higher percentage of missing insurance data in time period $\mathrm{A}$. The higher insured rate in time period $\mathrm{B}$ was mainly due to an increase in MediCal-covered patients.

The distribution of recommended referral intervals was similar in the two time periods: on average, immediate to 2 weeks, 22/408 (5\%); 1 month, 358/408 (88\%); 2-3 months, 23/408 (6\%); and 6 months, 5/408 (1.2\%). 
Table 1 Baseline patient characteristics by time period

\begin{tabular}{|c|c|c|c|c|c|c|c|}
\hline \multirow[b]{3}{*}{ Characteristics } & \multirow{2}{*}{\multicolumn{2}{|c|}{ All }} & \multicolumn{4}{|c|}{ Time period } & \multirow[b]{3}{*}{ P value* } \\
\hline & & & \multicolumn{2}{|c|}{$\begin{array}{l}\text { A } \\
2012-2014\end{array}$} & \multicolumn{2}{|c|}{$\begin{array}{l}\text { B } \\
2015-2017\end{array}$} & \\
\hline & $\mathbf{n}$ & $\%$ & $\mathbf{n}$ & $\%$ & $\mathbf{n}$ & $\%$ & \\
\hline Total & 408 & 100.0 & 147 & 36.0 & 261 & 64.0 & \\
\hline Age group & & & & & & & 0.0154 \\
\hline $18-49$ & 145 & 35.5 & 64 & 43.5 & 81 & 31.0 & \\
\hline $60+$ & 99 & 24.3 & 26 & 17.7 & 73 & 28.0 & \\
\hline Gender & & & & & & & 0.8592 \\
\hline Female & 203 & 49.8 & 74 & 50.3 & 129 & 49.4 & \\
\hline Male & 205 & 50.2 & 73 & 49.7 & 132 & 50.6 & \\
\hline Ethnicity & & & & & & & 0.0225 \\
\hline$<$ SNPDR & 210 & 51.5 & 63 & 42.9 & 147 & 56.3 & \\
\hline SNPDR & 61 & 15.0 & 15 & 10.2 & 46 & 17.6 & \\
\hline PDR & 137 & 33.6 & 69 & 46.9 & 68 & 26.1 & \\
\hline Clinically significant macular edema & & & & & & & 0.0295 \\
\hline No & 77 & 18.9 & 36 & 24.5 & 41 & 15.7 & \\
\hline Yes & 331 & 81.1 & 111 & 75.5 & 220 & 84.3 & \\
\hline Comorbidity index & & & & & & & 0.4721 \\
\hline 0 & 351 & 86.0 & 127 & 86.4 & 224 & 85.8 & \\
\hline $1+$ & 52 & 12.7 & 17 & 11.6 & 35 & 13.4 & \\
\hline No & 120 & 29.4 & 40 & 27.2 & 80 & 30.7 & \\
\hline Yes & 281 & 68.9 & 101 & 68.7 & 180 & 69.0 & \\
\hline Unknown & 7 & 1.7 & 6 & 4.1 & 1 & 0.4 & \\
\hline Type of diabetes & & & & & & & 0.5225 \\
\hline I & 24 & 5.9 & 8 & 5.4 & 16 & 6.1 & \\
\hline II & 381 & 93.4 & 137 & 93.2 & 244 & 93.5 & \\
\hline Unknown & 3 & 0.7 & 2 & 1.4 & 1 & 0.4 & \\
\hline Diabetes duration & & & & & & & 0.0884 \\
\hline $0-5$ years & 88 & 21.6 & 25 & 17.0 & 63 & 24.1 & \\
\hline $6-10$ years & 86 & 21.1 & 36 & 24.5 & 50 & 19.2 & \\
\hline $10+$ years & 229 & 56.1 & 86 & 58.5 & 143 & 54.8 & \\
\hline Unknown & 5 & 1.2 & 0 & 0.0 & 5 & 1.9 & \\
\hline $\mathrm{HbA1c}$ value & & & & & & & 0.146 \\
\hline$<6.0$ & 15 & 3.7 & 9 & 6.1 & 6 & 2.3 & \\
\hline $6.0-9.0$ & 147 & 36.0 & 52 & 35.4 & 95 & 36.4 & \\
\hline$>9.0$ & 164 & 40.2 & 53 & 36.1 & 111 & 42.5 & \\
\hline Unknown & 82 & 20.1 & 33 & 22.4 & 49 & 18.8 & \\
\hline
\end{tabular}

Continued 


\begin{tabular}{|c|c|c|c|c|c|c|c|}
\hline \multirow[b]{3}{*}{ Characteristics } & \multirow{2}{*}{\multicolumn{2}{|c|}{ All }} & \multicolumn{4}{|c|}{ Time period } & \multirow[b]{3}{*}{ P value* } \\
\hline & & & \multicolumn{2}{|c|}{$\begin{array}{l}\text { A } \\
2012-2014\end{array}$} & \multicolumn{2}{|c|}{$\begin{array}{l}\text { B } \\
2015-2017\end{array}$} & \\
\hline & $\mathbf{n}$ & $\%$ & $\mathbf{n}$ & $\%$ & $\mathbf{n}$ & $\%$ & \\
\hline Insured category & & & & & & & $<0.001$ \\
\hline Insured & 223 & 54.7 & 55 & 37.4 & 168 & 64.4 & \\
\hline Uninsured (charity, self-pay, uninsured) & 79 & 19.4 & 47 & 32.0 & 32 & 12.3 & \\
\hline Unknown & 106 & 26.0 & 45 & 30.6 & 61 & 23.4 & \\
\hline Insurance type & & & & & & & $<0.001$ \\
\hline Medicare & 27 & 6.6 & 6 & 4.1 & 21 & 8.0 & \\
\hline MediCal & 151 & 37.0 & 35 & 23.8 & 116 & 44.4 & \\
\hline Charity & 3 & 0.7 & 0 & 0.0 & 3 & 1.1 & \\
\hline Self-pay/uninsured & 76 & 18.6 & 47 & 32.0 & 29 & 11.1 & \\
\hline Unknown & 106 & 26.0 & 45 & 30.6 & 61 & 23.4 & \\
\hline Private & 6 & 1.5 & 4 & 2.7 & 2 & 0.8 & \\
\hline MISP & 39 & 9.6 & 10 & 6.8 & 29 & 11.1 & \\
\hline
\end{tabular}

${ }^{*} \mathrm{P}$ value from $\chi^{2}$ test or Fisher's exact test for difference of patient characteristics in two time periods.

MISP, Medically Indigent Services Program; PDR, proliferative diabetic retinopathy; SNPDR, severe non-proliferative diabetic retinopathy.

\section{Adherence rates}

Adherence rates for keeping a first ophthalmology appointment

Very few patients kept their first ophthalmology appointment within the recommended referral interval: 8/147 $(5 \%)$ in time period $\mathrm{A} ; 12 / 261(5 \%)$ in time period $\mathrm{B}$. For keeping first appointments within twice the recommended interval, only 19/147 (14\%) in period A and $42 / 261$ (16\%) in period B adhered. When the latter results are stratified by the length of the recommended interval, those recommended for $<2$ weeks and those for 3 months had a higher average adherence rate, 10/22 $(45 \%)$ and $6 / 23$ (26\%), respectively, compared with those recommended for a 1 month interval, 44/358 $(11 \%)(\mathrm{p}<0.0001)$.

For keeping a first ophthalmology appointment within 1 year of the DRS, the adherence rates were 70/147 $(48 \%)$ in time period $\mathrm{A}$ and $147 / 261(56 \%)$ in time period $\mathrm{B}(\mathrm{p}<0.05)$. An additional $13 / 147(9 \%)$ in time

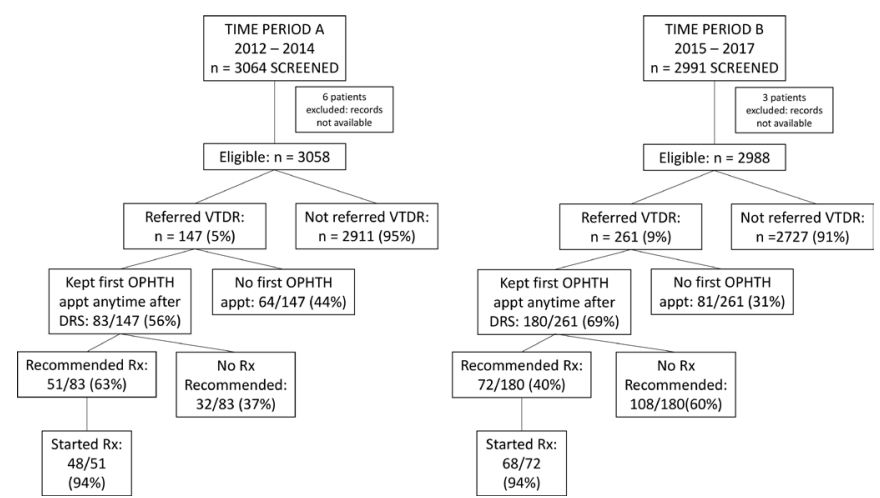

Figure 1 Diagram showing the original study sample and the major findings of the study. appt, appointment; DRS, diabetic retinopathy screening; OPHTH, ophthalmology; VTDR, vision-threatening diabetic retinopathy. period A and 33/261 (13\%) in time period B kept a first ophthalmology appointment beyond 1 year of the DRS.

Figure 2 shows the adherence data over time plotted as time-to-event estimates (Kaplan-Meier curves), where the 'event' was keeping a first ophthalmology appointment. The upper curve (2015-2017) shows a more rapid development of the event than the lower curve (2012-2014), $\log$ rank $\mathrm{p}=0.0109$.

Only $17 / 195(9 \%)$ of patients failing to keep an ophthalmology appointment within 1 year of the initial DRS had a repeat screening within 2 years of the DRS. Among the patients with repeat screening, 14/17 (82\%) were again referred to ophthalmology for VTDR, and of

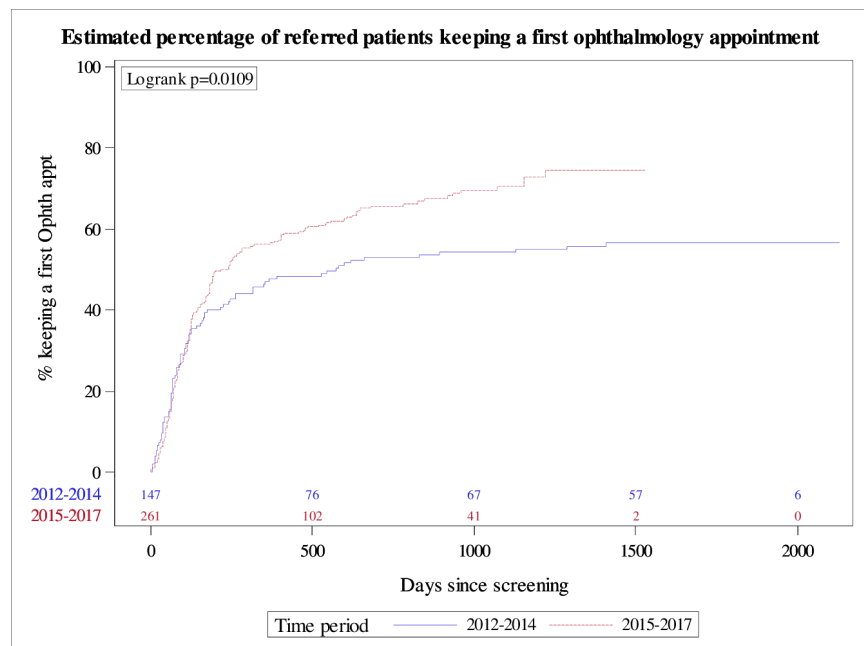

Figure 2 Time-to-event estimates: Kaplan-Meier curves comparing time period A, 2012-2014 (lower curve), with time period B, 2015-2017 (upper curve). The 'event' was keeping a first ophthalmology appointment. The number of subjects at risk for each time period is shown above the $\mathrm{x}$-axis. 
A A Overall Adherence with Follow-up After Rx Started

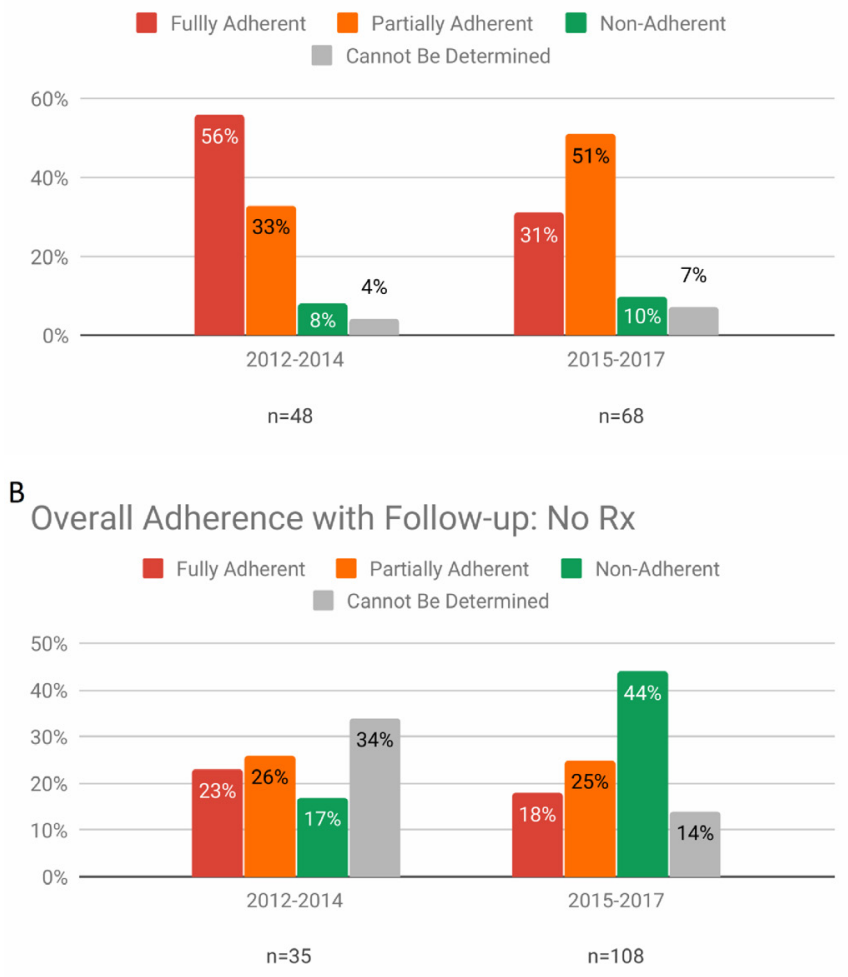

Figure 3 (A) Proportion of patients starting treatment who adhered to post-treatment follow-up recommendations after treatment was started. (B) Proportion of patients keeping a first ophthalmology appointment and NOT recommended for treatment, who adhered to follow-up recommendations in the eye clinic.

those, 6/14 (43\%) kept a first ophthalmology appointment within 1 year. Therefore, the 'recapture rate' was only $6 / 195(3 \%)$.

There were six patients who died during the follow-up period; $4 / 6$ had kept a first ophthalmology appointment within 1 year of the DRS. Of the two patients without a first ophthalmology appointment, one died $2^{1 / 2}$ years after the DRS, and the other died 8 months after the DRS.

The relationship between the adherence rates and various patient characteristics is presented in detail in online supplementary appendix B.

\section{Treatment (Rx) recommendation status; adherence to initiation of treatment and to post-treatment follow-up Treatment recommendation status}

Among the patients keeping a first ophthalmology appointment in time period A, 51/83 (61\%) were recommended for treatment, compared with only $72 / 180(40 \%)$ in time period $B(p=0.0012)$, with an overall average for both time periods of $47 \%$. There was no significant difference in retinopathy distribution between the two time periods among patients recommended for treatment.

The types of treatment recommended included focal laser, panretinal photocoagulation (PRP), intravitreal injections with anti-vascular endothelial growth factor (anti-VEGF agents), and vitrectomy surgery. Sixty-seven of $123(54 \%)$ patients had a combination of treatment types recommended, and 56/123 (46\%) a single treatment type. PRP and intravitreal anti-VEGF injections comprised the most common combination, 35/67 (52\%), as well as being the most common single treatment types, $25 / 56(45 \%)$ and 24/56 (43\%), respectively.

Overall, $137 / 263(52 \%)$ of the referred patients keeping a first ophthalmology appointment were thought not to need treatment. Of these, 55/137 (40\%) were diagnosed with VTDR by ophthalmological examination, but were not recommended for treatment. The decision not to treat at that time had been based on one or more factors: (1) SNPDR, and no diabetic macular edema; (2) prior treatment with PRP, and no additional treatment needed; (3) macular edema present, but not involving or threatening the central macula. The rest, 79/137 (58\%), were found to have retinopathy less severe than VTDR by ophthalmological examination.

Among the referred patients with VTDR who kept a first ophthalmology appointment, the ophthalmological examination found a greater proportion of patients with moderate non-proliferative diabetic retinopathy and no CSME in time period B, 63/180 (37\%), compared with $18 / 83(22 \%)$ in time period A; none of these patients were recommended for treatment. Conversely, there were more patients with PDR in time period A $(37 / 83$ $(45 \%))$ than in time period B (58/180 (32\%)); $92 \%$ of these were recommended for treatment in time period A and $72 \%$ in time period $\mathrm{B}$.

\section{Adherence to initiation of treatment}

One hundred and sixteen out of 123 (94\%) patients recommended for treatment initiated the treatment. Treatment was usually started in the ophthalmology clinic the same day that it was recommended.

\section{Adherence to post-treatment follow-up}

The rate of complete adherence to post-treatment follow-up was higher in time period A, 27/48 (56\%), than in time period $\mathrm{B}, 21 / 68(31 \%)(\mathrm{p}=0.006)$, although it was suboptimal in both (figure $3 \mathrm{~A}$ ). The rates of partial adherence were not statistically significantly different by time period: time period A, 16/48 (33\%), and time period B, 34/68 (51\%). Non-adherence was relatively low in both time periods $(8 \%, 10 \%)$ as were the proportion of patients for whom adherence was indeterminate $(4 \%$, $7 \%)$.

\section{Adherence to follow-up visits in the ophthalmology clinic for} patients not recommended for treatment

Most patients not recommended for treatment were advised to return to the ophthalmology clinic for repeat evaluations. The rates of adherence with follow-up were generally low (figure 3B). In time period $\mathrm{A}$, almost half of the patients were either non-adherent, 6/35 (17\%), or partially adherent, $9 / 35$ (26\%); in time period $\mathrm{B}$, a 
greater proportion of patients were either non-adherent, $47 / 108(44 \%)$, or partially adherent, 27/108 (25\%) $\left(\chi^{2}\right.$ test $p$ value $\left.=0.0108\right)$. The proportion of patients for whom the adherence was indeterminate was relatively high in both time periods ( $34 \%$ and $14 \%$ ).

Comparison of adherence to follow-up among all patients recommended for treatment $(\mathrm{Rx})$ with all those not recommended (no Rx) showed that the complete compliance rate in the $\mathrm{Rx}$ group, 48/109 (44.0\%), was significantly greater than in the no-Rx group, 27/116 $(23.3 \%)(\mathrm{p}=0.0010)$.

\section{Multivariate Cox proportional hazards model}

The HRs for keeping a first ophthalmology appointment, derived from the multivariate Cox proportional hazards model, are reported in online supplementary appendix B. Patients in time period B were more likely to be adherent compared with patients in time period A $(\mathrm{HR}=1.40,95 \%$ CI 1.05 to $1.87, \mathrm{p}=0.023)$. Other covariates that were significantly associated with greater adherence were Hispanic patients, patients with PDR (compared with less than SNPDR), patients with one or more comorbid conditions (compared with no comorbid condition) and diabetes duration 0-5years (compared with $6-10$ years). Health insurance status was not significantly related to adherence.

\section{DISCUSSION}

While telemedicine-based DRS can dramatically increase the screening rate by moving the screening locus from the specialty eye care setting to the primary care setting, the management of patients identified with VTDR through DRS still requires a referral out to a specialist. Therefore, one would expect that many of the same barriers that impeded DRS by referral would also impede treatment by referral. The major difference from the healthcare delivery and quality improvement viewpoints is that with successful DRS, at least we know who the patients are who need to be 'moved', and the challenge becomes how to encourage them to move, not how to find them.

Although several published studies have reported adherence data for ophthalmic follow-up after DRS in the primary care setting, they have usually been restricted to tracking the first ophthalmology visits and not the subsequent treatment and post-treatment visits. ${ }^{416-18}$ There are a number of studies conducted entirely in the ophthalmology setting that track the adherence of patients with VTDR to various modalities of treatment. ${ }^{19-21}$ The present study appears to be the first to report the results of tracking patients from the primary care screening setting all the way through the specialty referral and subsequent treatment and post-treatment phases.

Overall, about $50 \%$ of patients failed to keep any first ophthalmology appointment within 1 year of the DRS, a deficit that can lead to clinically significant vision loss, especially with PDR. ${ }^{22}$ No formal system was in place to identify and rescreen patients who failed to keep an ophthalmology appointment. Rather, there was a general policy in the primary care clinics to offer DRS to any patient who had not been screened for at least 12 months or who had not seen an eye care provider for at least 2 years. However, the rate of rescreening for the nonadherent patients was very low, underscoring the need for a more robust tracking and recall system for patients failing to keep a first ophthalmology appointment.

The records showed that only six patients died during the observed time frame after the DRS. The effect of death on adherence rates to keeping a first ophthalmology appointment within 1 year was negligible; four patients adhered, one patient did not adhere, but died beyond the 1 year post-DRS period, while only one patient died within the 1 year post-DRS period.

There were differences in adherence rates to keeping a first ophthalmology appointment depending on the recommended referral interval. Higher adherence rates for keeping a first ophthalmology appointment within twice the recommended interval were found both for patients recommended for referral within 2 weeks of the DRS $(45 \%)$ or within 3 months $(26 \%)$ compared with those with a 1 month interval $(11 \%)$. The higher adherence rate for the $\leq 2$ weeks' interval can be attributed to an informal 'fast track workaround', in which a primary care staff directly contacted the ophthalmology clinic, either by telephone or in person, to facilitate the referral of the most urgent patients. ${ }^{23}$ The higher adherence for the 3 months' interval can probably be explained simply by the longer interval, giving the patients more time to adhere to the recommendation.

Overall, patients in time period B went sooner to their first ophthalmology appointment than those in time period A, according to the Kaplan-Meier time-to-event estimates. This is reinforced by finding an increased likelihood of keeping the first ophthalmology appointment among patients in time period $\mathrm{B}$ by multivariate regression analysis (online supplementary appendix B). These results could relate to more efficient referral processes with the EMR. However, finding that almost $30 \%$ of the year 2017 patients who failed to keep a first appointment within 1 year showed no evidence of a formal referral to ophthalmology in the EMRs (online supplementary appendix A) demonstrates that while the EMR can improve communications between primary care providers and specialists and improve the quality of care, ${ }^{15}$ fail-safe processes for making and tracking referrals are still needed.

Other patient characteristics significantly associated with a higher likelihood of adherence were Hispanic ethnicity, a comorbidity index of 1 or more, and the diagnosis of PDR. Having health insurance did not significantly correlate with adherence likelihood (online supplementary appendix B). The latter finding is consistent with reports elsewhere that the increased coverage with Medicaid, achieved after the introduction of the Affordable Care Act, did not, by itself, lead to increased utilization of certain screening services. ${ }^{24} 25$ 
A higher percentage of patients with kept first ophthalmology appointments were recommended for treatment in time period A than in time period B. This could be due to the higher prevalence of patients with PDR in the time period A sample, which, in turn, could indicate a backlog of previously undetected patients with PDR in the earlier phase of the retinal screening program. ${ }^{26}$

Once treatment was recommended, a very high proportion $(94 \%)$ of patients initiated the treatment. Post-treatment follow-up visits were always scheduled to evaluate the effects of treatment, and to consider adding treatment. This was especially important for patients receiving intravitreal anti-VEGF treatment, for whom at least three to four treatment sessions in a series were frequently planned over a period of 3-6 months.

The proportion of patients who initiated treatment and then adhered to all post-treatment follow-up visits (within 6 months of the recommended visit date) was significantly greater in time period A than in time period B. This could be an artifact relating to greater difficulty in identifying missed visits in the paper charts. While more missed visits have been reported among patients receiving PRP compared with anti-VEGF injections, ${ }^{19}$ our sample was not large enough to stratify the results by individual treatment types.

Among the patients not recommended for treatment, the non-adherence rates for follow-up were significantly higher in time period B compared with A. However, since the proportion of patients for whom adherence was indeterminate was significantly higher in time period A compared with $\mathrm{B}$, the validity of comparing adherence rates by time period is questionable.

Comparison between patients recommended for treatment $(\mathrm{Rx})$, with those not recommended for treatment (no Rx), showed significantly poorer adherence with recommended follow-up visits in the no-Rx group. Recommended follow-up intervals were usually longer in the no-Rx group, up to 1 year in many cases. With the longer intervals, a specific appointment may not have been made during the initial ophthalmology visit. In addition, there was probably a lesser sense of urgency imparted to these patients because of the low immediate threat to vision. Again, the relatively high indeterminate rate in the no-Rx group limits the validity of this comparison.

The results of this study indicate that the EMR provides more complete data on the referral process and on adherence characteristics than the paper charts. This included dates of formal referral to ophthalmology, attendance at other outpatient clinics, and missed follow-up visits, data for which were often not available or not reliable in paper charts. (See online supplementary appendix A.)

There are limits to the generalizability of the findings from this study. The present study was conducted in a primary care clinic of a safety net hospital serving a mostly low-income population. The insurance payer mix of the subjects in the study was weighted toward safety net, publicly funded insurance, specifically MediCal and
MISP. In settings where there is a preponderance of private insurance payers and Medicare, the adherence characteristics would conceivably be better, as has been reported for the rates of DRS itself. ${ }^{27}$ The ophthalmology services at RUHS-MC were located in the same building as the primary care clinics. In primary care settings where ophthalmology is located off-site, the adherence characteristics might understandably be worse.

The present study was also limited by the lack of availability of data from patients who may have been evaluated and treated elsewhere for their VTDR. It is likely that at least some of the non-adherence occurred for that reason. As example, we found that 16/114 (14\%) of patients referred for VTDR in time period B who failed to keep a first ophthalmology appointment within 1 year had no RUHS outpatient visit beyond 6 months after the date of the DRS. Tracking of patients like this in future studies would provide a more complete picture of the true adherence rates. Conversely, most patients who were nonadherent or partially adherent to eye care recommendations continued to receive non-ophthalmic medical care at RUHS. This suggests that with an enhanced tracking and recall system, improved adherence to eye care may be achievable through targeted interventions.

Our study did not look at adherence to treatment and follow-up in the ophthalmology clinic for patients with other chronic eye diseases such as glaucoma. It is possible that the adherence deficits found for patients with diabetes and VTDR are a more generalized problem for patients with chronic eye disease, or, as seems likely, for the safety net population as a whole. While addressing the multifactorial causes for adherence deficits in the safety net system is daunting, collecting adherence data, such as was done retrospectively in the present study, is an essential step in improving the quality of care. Otherwise, the problem cannot be defined or may not even be recognized.

As a result of our findings, the primary care clinics at RUHS-MC have redesigned the workflow for DRS and the referral process. They have begun to provide results of DRS immediately to the patients before they leave the clinic. This has been coupled with enhanced patient engagement to explain the risk of vision loss from untreated VTDR and how to prevent it, as well as provision of prompt appointments to the ophthalmology clinic for the more urgent referrals ( $\leq 1$ month). An improved tracking and recall system for patients not keeping a first ophthalmology appointment has also been instituted. The adherence rates of patients enrolled in this new program will be compared with the results reported in the present study in an ongoing prospective study.

\section{Author affiliations}

${ }^{1}$ University of California Berkeley School of Optometry, Berkeley, California, USA

${ }^{2}$ EyePACS, Santa Cruz, California, USA

${ }^{3}$ Family Medicine, Riverside University Health System, Riverside, California, USA

${ }^{4}$ Advanced Clinical, Chicago, Illinois, USA

${ }^{5}$ Medicine, University of California Irvine College of Medicine, Irvine, California, USA

${ }^{6}$ Epidemiology, University of California Irvine, Irvine, California, USA

${ }^{7}$ Google, Mountain View, California, USA 
Acknowledgements Dr Geoffrey Leung provided administrative support for the study. Divya Reddy, Rosio Sandoval, Norma Farias-Zambrano, and Dalia Parias participated in the record review. Dr Taras Litvin helped design the protocol.

Contributors GB designed the study protocol, researched the data, contributed to discussion, drafted the manuscript, and reviewed and edited the manuscript. JAC helped design the study protocol, researched the data, contributed to discussion, and reviewed and edited the manuscript. PC and AL researched the data, contributed to discussion, and reviewed and edited the manuscript. SF, ERP GW, and MK contributed to discussion, and reviewed and edited the manuscript. JC and $L J$ conducted the statistical analyses and reviewed and edited the manuscript. $\mathrm{GB}$ is the guarantor of this work, and as such had full access to all the data in the study and takes responsibility for the integrity of the data and the accuracy of the data analysis. All authors have read and approved the paper.

Funding This study was supported by Google and EyePACS.

Competing interests GB, JAC and PC declared competing interests based on EyePACS affiliation. SF, ERP, and GW declared competing interests based on affiliation with Google.

Patient consent for publication Not required.

Ethics approval This study was approved by the Riverside University Health System IRB Committee (No. 824116-9). All patient data were deidentified, and patient consent for this retrospective study was not required by the IRB.

Provenance and peer review Not commissioned; externally peer reviewed.

Data availability statement Data are available upon reasonable request. The data are deidentified participant data, available from GB; visionforall2020@gmail.com.

Open access This is an open access article distributed in accordance with the Creative Commons Attribution Non Commercial (CC BY-NC 4.0) license, which permits others to distribute, remix, adapt, build upon this work non-commercially, and license their derivative works on different terms, provided the original work is properly cited, appropriate credit is given, any changes made indicated, and the use is non-commercial. See: http://creativecommons.org/licenses/by-nc/4.0/.

ORCID iDs

George Bresnick http://orcid.org/0000-0001-9604-5403

Jorge A Cuadros http://orcid.org/0000-0002-7804-5386

Mahbuba Khan http://orcid.org/0000-0002-0045-5107

Sybille Fleischmann http://orcid.org/0000-0003-4810-4741

Gregory Wolff http://orcid.org/0000-0001-7703-8654

Jenny Chang http://orcid.org/0000-0002-6755-8928

Luohua Jiang http://orcid.org/0000-0002-2281-7260

Pablo Cuadros http://orcid.org/0000-0001-5717-1204

Elin Rønby Pedersen http://orcid.org/0000-0002-3023-8939

\section{REFERENCES}

1 Scanlon $\mathrm{PH}$. The English national screening programme for diabetic retinopathy 2003-2016. Acta Diabetol 2017;54:515-25.

2 Daskivich LP, Vasquez C, Martinez C, et al. Implementation and evaluation of a large-scale teleretinal diabetic retinopathy screening program in the Los Angeles County department of health services. JAMA Intern Med 2017;177:642-9.

3 Mansberger SL, Sheppler C, Barker G, et al. Long-term comparative effectiveness of telemedicine in providing diabetic retinopathy screening examinations: a randomized clinical trial. JAMA Ophthalmol 2015;133:518-25.

4 Jani PD, Forbes L, Choudhury A, et al. Evaluation of diabetic retinal screening and factors for ophthalmology referral in a telemedicine network. JAMA Ophthalmol 2017;135:706-14.

5 Vujosevic S, Benetti E, Massignan F, et al. Screening for diabetic retinopathy: 1 and 3 nonmydriatic 45 -degree digital fundus photography vs 7 standard early treatment diabetic retinopathy study fields. Am J Ophthalmol 2009;148:111-8.

6 Smith-Morris C, Bresnick GH, Cuadros J, et al. Diabetic retinopathy and the cascade into vision loss. Med Anthropol 2020;39:109-122.
7 Ferris FL. How effective are treatments for diabetic retinopathy? JAMA 1993;269:1290-1.

8 National Eye Institute. People with diabetes can prevent vision loss. Available: https://www.nei.nih.gov/sites/default/files/2019-06/ diabetes-prevent-vision-loss.pdf

9 Mehrotra A, Forrest CB, Lin CY. Dropping the baton: specialty referrals in the United States. Milbank Q 2011;89:39-68.

10 Tseng VL, Greenberg PB, Scott IU, et al. Compliance with the American academy of ophthalmology preferred practice pattern for diabetic retinopathy in a resident ophthalmology clinic. Retina 2010;30:787-94.

11 Quade R. Evaluation of the expanding access to diabetic retinopathy screening initiative. evaluation report. Oakland, CA: California Healthcare Foundation, 2011.

12 Thompson AC, Thompson MO, Young DL, et al. Barriers to follow-up and strategies to improve adherence to appointments for care of chronic eye diseases. Invest Ophthalmol Vis Sci 2015;56:4324-31.

13 Cuadros J, Bresnick G. EyePACS: an adaptable telemedicine system for diabetic retinopathy screening. J Diabetes Sci Technol 2009;3:509-16.

14 Wong TY, Sun J, Kawasaki R, et al. Guidelines on diabetic eye care: the international council of ophthalmology recommendations for screening, follow-up, referral, and treatment based on resource settings. Ophthalmology 2018;125:1608-22.

15 Stille CJ, McLaughlin TJ, Primack WA, et al. Determinants and impact of generalist-specialist communication about pediatric outpatient referrals. Pediatrics 2006;118:1341-9.

16 Keenum Z, McGwin G, Witherspoon CD, et al. Patients' adherence to recommended follow-up eye care after diabetic retinopathy screening in a publicly funded County clinic and factors associated with follow-up eye care use. JAMA Ophthalmol 2016;134:1221-8.

17 Negretti GS, Amin R, Webster L, et al. Does delay in referral of proliferative diabetic retinopathy from the diabetic eye screening programme lead to visual loss? Eye 2016;30:873-6.

18 Date RC, Shen KL, Shah BM, et al. Accuracy of detection and grading of diabetic retinopathy and diabetic macular edema using teleretinal screening. Ophthalmol Retina 2019;3:343-9.

19 Obeid A, Gao X, Ali FS, et al. Loss to follow-up in patients with proliferative diabetic retinopathy after panretinal photocoagulation or intravitreal anti-VEGF injections. Ophthalmology 2018;125:1386-92.

20 Weiss M, Sim DA, Herold T, et al. Compliance and adherence of patients with diabetic macular edema to intravitreal antivascular endothelial growth factor therapy in daily practice. Retina 2018;38:2293-300.

21 Angermann R, Rauchegger T, Nowosielski Y, et al. Treatment compliance and adherence among patients with diabetic retinopathy and age-related macular degeneration treated by anti-vascular endothelial growth factor under universal health coverage. Graefes Arch Clin Exp Ophthalmol 2019;257:2119-25.

22 Photocoagulation treatment of proliferative diabetic retinopathy. clinical application of diabetic retinopathy study (DRS) findings, DRS report number 8 . the diabetic retinopathy study Research Group. Ophthalmology 1981;88:583-600.

23 Bouskill K, Smith-Morris C, Bresnick G, et al. Blind spots in telemedicine: a qualitative study of staff workarounds to resolve gaps in diabetes management. BMC Health Serv Res 2018;18:617.

24 Chen EM, Armstrong GW, Cox JT, et al. Association of the affordable care act medicaid expansion with dilated eye examinations among the United States population with diabetes. Ophthalmology 2019;6420:32056.

25 Alharbi AG, Khan MM, Horner R, et al. Impact of medicaid coverage expansion under the affordable care act on mammography and Pap tests utilization among low-income women. PLoS One 2019;14:e0214886

26 Scanlon P. An evaluation of the effectiveness and cost-effectiveness of screening for diabetic retinopathy by digital imaging photography \& technician ophthalmoscopy \& the subsequent change in activity, workload and costs of new diabetic Ophthalmology referrals. London, 2005.

27 Shi Q, Zhao Y, Fonseca V, et al. Racial disparity of eye examinations among the U.S. working-age population with diabetes: 2002-2009. Diabetes Care 2014;37:1321-8. 